\title{
Family Medicine and Community Health \\ Engaging diverse community members to enhance analysis and interpretation: processing qualitative interview data
}

\author{
Sarah E Brewer (D) ,1,2 Jean Scandlyn ${ }^{3,4}$
}

To cite: Brewer SE, Scandlyn J. Engaging diverse community members to enhance analysis and interpretation: processing qualitative interview data. Fam Med Com Health 2022;10:e001235. doi:10.1136/ fmch-2021-001235

\section{Check for updates}

(C) Author(s) (or their employer(s)) 2022. Re-use permitted under CC BY-NC. No commercial re-use. See rights and permissions. Published by BMJ.

${ }^{1}$ Family Medicine, University of Colorado - Anschutz Medical Campus, Aurora, Colorado, USA ${ }^{2}$ ACCORDS, University of Colorado - Anschutz Medical Campus, Aurora, Colorado, USA ${ }^{3}$ Health and Behavioral Sciences, University of Colorado Denver, Denver, Colorado, USA ${ }^{4}$ Anthropology, University of Colorado Denver, Denver, Colorado, USA

Correspondence to Dr Sarah E Brewer; Sarah.brewer@cuanschutz.edu

\section{ABSTRACT}

As the USA becomes more diverse, the inclusion of patients from diverse backgrounds in research becomes ever more important to ensuring a complete understanding of the patient experience in primary care. Language and cultural barriers are important areas in which researchers face substantial challenges. Primary care researchers need tools and approaches to include diverse communities in qualitative interviews. Here, we describe one way primary care researchers can apply an adapted, engaged transcription and interpretation method in qualitative research to improve retention of nuance and meaning across language and cultures, specifically with nonEnglish, non-Spanish-speaking resettled refugees. We also discuss how the approach provided additional information that increased the validity of interpretation and analysis and improved the retention of nuance in a qualitative primary care study. The methodological and practical value, scope of application and potential limitations and improvements of this method through future research are addressed.

\section{INTRODUCTION}

The USA becoming ever more diverse. ${ }^{12}$ In 2019, the Census Bureau reported that over $40 \%$ of the US population was either Hispanic or non-white and over $20 \%$ of families reported speaking a language other than English at a home. ${ }^{3}$ In some 'superdiverse' communities-communities with multiple intersecting layers of diversity by characteristics such as culture, language, ethnicity, race, country of origin and immigration status, ${ }^{12}$ these numbers are much higher. ${ }^{4}$ For example, communities and neighbourhoods that accept arriving refugees, asylum-seekers, and other immigrants, which Robinson termed 'arrival zones' tend to have high levels of heterogeneity and complexity in the make-up of the community. ${ }^{5}$ This 'superdiversity' creates additional challenges when it comes to inclusion in research. For example, whereas in the US Spanish speakers are included in research and many research instruments are available in Spanish, this is typically not the case for other languages.
The field of primary care seeks to treat patients of all backgrounds in the context of their families and communities. The inclusion of patients from these diverse backgrounds in research projects becomes ever more important to ensuring a complete understanding of the patient experience in primary care. In addition, representation in research helps the discipline as a whole and individual practices to be responsive to the needs of all patients. However, due to various challenges, primary care and family medicine have not met their potential in including diverse patient representation in research.

Language and cultural barriers are important areas in which research faces substantial challenges in effectively including diverse communities in health research. This becomes especially true for qualitative research in which the inclusion of cultural and language diversity in study populations can introduce additional complexity and cost to the conduct of a study. For example, adding a language group can introduce prohibitive transcription and translation costs to data processing. These data processing steps can also result in the loss of cultural nuances that are difficult or impossible to translate precisely through traditional transcription or translation mechanisms, especially when contracted to professionals who are otherwise unfamiliar with the topic of the research. These logistical, cost and meaning retention challenges can result in eligibility criteria that exclude portions of a patient population, such as 'Patient must be able to respond in English or Spanish.' These challenges can be daunting to researchers, causing them to shy away from such research endeavours. These exclusions from studies result in the exclusion of smaller minority communities from primary care research.

When language and cultural diversity are included in primary care research, researchers can take several different approaches. The standard approach to translation working in health-related survey research is translation and 
back translation. Critics of this method note that it is often used mechanically, translating survey questions word-forword; thus, researchers may substitute words without direct equivalence in the other language, not recognising that the concepts underlying the words may not be shared in that cultural context. ${ }^{6}$ Anthropologists have a long tradition of translating language and cultural concepts and context. They often rely on key informants, native speakers and community members who can translate language and cultural context. ${ }^{7}$ However, most anthropologists work with one population for an extended period and have a high level of fluency in the target language. While this model of cultural translation has its own limitations, ${ }^{89}$ it can be applied in work with super diverse populations by working closely with native speakers and community members who understand the goals of the research and receive training in cross-cultural research. ${ }^{6710}$ To meet these needs, primary care and family medicine researchers need tools and approaches to engage and include diverse communities in primary care research, especially in qualitative interviews. Here, we describe one way primary care researchers can apply an adapted, engaged transcription and interpretation method in qualitative research to improve retention of nuance and meaning across language and cultures. Specifically, we outline a method for engaging community members, in this case refugees, as members of the research team and specifically in transcription and notation of in-depth interviews with non-English, non-Spanish speakers. ${ }^{11-13}$ Next, we will explain how this method added value to our approach, compared with traditional qualitative data processing and analysis. We discuss how this approach improved the retention of nuance and meaning across language and culture in one qualitative primary care study. Finally, we will discuss the methodological and practical value, scope of application, and potential limitations and improvements of this method through future research.

\section{Engaged qualitative approach in superdiverse community Case overview}

The primary goal of the project in which this method was developed was to understand primary care experiences among young adult resettled refugees, including the barriers and facilitators to accessing care, relationships with primary care providers and cultural perceptions of primary care as distinct from other types of healthcare. The study was conducted in the Denver metropolitan area of Colorado. The sampling frame was any resettled refugee ages 18-29 who had been in the USA at least 2 years and had a country of origin of Burma (Myanmar), Bhutan, Iraq, Syria or Somalia. This sampling frame included five of the most common countries of origin resettled to the study setting over the 5 years prior to the study as well as a mix of visa types (eg, refugee, special immigrant visas), education levels in the country of origin, and resettlement situations (eg, short-term camps, protracted refugee camp situations).

Twenty-three young adult refugees participated in interviews and their average age was 24.7 years. Five participants reported their country of origin was Bhutan; five were from Burma; five were from Somalia, four were from Iraq and four were from Syria. Forty-four per cent of participants were male and $48 \%$ were married. Level of educational attainment varied: $39 \%$ reported less than a high school education, $39 \%$ earned a high school diploma, and 22\% completed an associate's or bachelor's degree. $87 \%$ reported living in an intermediate location for more than 3 years before being resettled to the USA. Forty-eight per cent reported living in a refugee camp. Thirty-nine per cent of participants were parents. Among those with children, they had an average of 2.6 children.

Participants provided verbal consent for participation and no identifying information was collected. All research assistants were provided with in-person interactive human subjects training with the director of regulatory affairs, following the community-engaged approach described by Westfall et al. ${ }^{14}$ They also received extensive training in semistructured interviewing from the authors.

\section{Research assistants}

Four research assistants who were also members of the refugee community were engaged as co-researchers and hired and trained to conduct semistructured qualitative interviews. All four research assistants spoke English fluently and were fluent in one or more of the most common languages in the target sample: Nepali Bhutanese, Burmese, Karen, Somali and Arabic; three also had formal training as interpreters. One research assistant was hired per each of these languages. Two research assistants served dual roles: one spoke both Burmese and Karen languages and had experience working with communities from both cultural backgrounds as a medical interpreter and community leader. Another research assistant spoke Arabic and had extensive experience working with both Iraqi and Syrian communities as a patient navigator. This approach of having one research assistant per language or culture group addressed pragmatic issues of community capacity for these roles in terms of time and specialised skills required to carry out the research tasks. The research assistants were engaged in the research process using elements of community-based participatory research. ${ }^{15}{ }^{16}$ While they were not engaged in the original design of the research study, once hired onto the study research assistants were partners and principles of colearning and co-ownership were applied to all aspects of the work.

\section{In-depth interviews}

In-depth narrative interviews were conducted with young adult resettled refugees to understand the experience of primary care and the healthcare system following resettlement to the USA. Recruitment took place in community settings and a combination of sampling methods, including venue based ${ }^{1718}$ and snowball sampling were applied. ${ }^{19}$ The interview schedule included seven questions to guide the narrative discussion; probing questions targeting barriers and facilitators of primary care use. To begin the conversation with the interviewer, the interview schedule incorporated a one-page timeline participants could use to gather their thoughts about their experiences with primary care. Participants also completed a brief demographics survey. Research 
assistants conducted interviews, which spanned between 40 and $60 \mathrm{~min}$, in the native languages of the participant and at locations agreed on by the interviewer and the participant, including local health clinics, libraries, community centres and participants' homes.

The narrative data collected in these semi-structured interviews was digitally audiorecorded and interviewers took field notes to capture non-verbal data. Finally, the research assistants who conducted interviews with non-English speakers transcribed the interviews into English and included notes in the transcripts comments from their field notes about nonverbal communication and cultural meanings that accompanied the verbatim words. These notes were formatted differently from the main text (eg, in italics or denoted with asterisks) to ensure clear demarcation from the text of the transcript. Complete data included an interview recording, an interview transcript, field notes, an interviewer debrief form (including key topics discussed, interviewer impressions, challenges with the interview or the interview guide, follow-up promised to the participant), a demographics survey and the timeline created by the interviewee.

\section{Translation and transcription}

Transcription was conducted using an adapted, pragmatic approach. The initial approach was to use voice-to-text transcription so that the interviewers could listen in the language of the interview, speak or interpret the conversation in English, and the software would transcribe the conversation as the research assistant spoke it aloud. Due to inconsistent accuracy of the software, and even worse accuracy when the speaker had a non-American or non-British accent, this approach proved inaccurate and time-consuming and was abandoned quickly. In collaboration with the research assistants, the team developed a process in which each interviewer listened to recordings of the interviews that they had conducted, usually at half speed or slower, while transcribing the conversation directly from the interviewee's native language into English. This approach leveraged the training and experience of each of the research assistants in interpretation, while bypassing the need to transcribe in the native language and then translate into English.

In addition to translating and transcribing the verbal data, interviewers concurrently reviewed their field notes while transcribing and added bracketed notations in the body of the transcripts, thus capturing non-verbal data, for example, (smiling and laughing), cultural context (such as gender norms or medical practice from country of origin), nuances of their translation of the conversation, eg (The participant said ' $\mathrm{X}$ ' in their native language, which could mean 'A' or 'B'. Based on the context and my understanding, I translated as 'B'.)), and their own reflections on the interview, for example, (participant seemed to disengage when addressing this topic) or (this topic has come up in other interviews, too). Embedding these notations resulted in a single document for each interview encounter that incorporated multiple types of data collected (verbal, non-verbal, cultural, contextual and translation). Other data sources, such as demographic surveys, field notes, participants timelines and interviewer debrief forms rounded out this qualitative dataset. These data were then transferred to ATLAS.ti V.8 (2018), which was used for data management and organisation throughout data analysis.

\section{Improved nuance and understanding}

This method for transcription and annotation of transcripts resulted in a richer dataset than traditional verbatim transcription in the participants' native language with subsequent translation of the transcript into English. In addition to clarifying cultural norms and references, this approach captured other nuances that would likely not have been possible through common translation and transcription procedures. For example, throughout data collection and analysis, it became clear that vocabulary for talking about healthcare sources was limited and that the context of the conversation between the participants and the interviewer was what differentiated to what type of clinical setting the participant referred. Transcripts from interviews with participants from all five sampled countries of origin included annotations such as:

- '*Here the participant is referring to a hospital'.

- 'By 'clinic' the participant means their doctor's office'.

- 'The participant said 'hospital', but they are talking about the (clinic name) refugee clinic.'

When discussing healthcare, participants tended to use the terms 'clinic' and 'hospital' interchangeably to refer to a place where they could seek healthcare. These were not used as specific terms that delineated the types of care that are available in different places. Rather, 'doctor's office,' 'clinic' and 'hospital' all simply referred to any and all healthcare facilities.

This lack of specificity in terminology regarding healthcare facilities has a few implications and potential meanings. First, this sometimes made it difficult to interpret the participants' meaning when discussing different sources of care and whether they were describing experiences of attempting to obtain primary care or other types of healthcare. Second, if the conflation of terminology for healthcare facilities is conceptual—not simply a function of difficulty in translating terms from participants' native languages - then this may contribute to further difficulty in distinguishing between where participants seek or need to seek different levels and types of care. For example, if young adult refugees believe that a hospital with inpatient facilities is the ideal place to seek all healthcare, then they will have difficulty engaging effectively with primary care providers who are located in standalone outpatient practices. This use of vocabulary and terminology is an important factor in understanding young adult refugees' interactions with healthcare and formed an underlying theme to the broader study.

\section{DISCUSSION}

Here, we have described a pragmatic, community-engaged approach to translating, transcribing and annotating 
qualitative interview data collected in superdiverse communities in order to provide additional information in transcripts and support interpretation and analysis of data. This approach had numerous advantages compared with standard approaches to multi-language data processing: overcoming drawbacks of back-translation, decreasing cost and increasing efficiencies, increasing inclusivity in the research process, and improving overall understanding for the researchers.

First, this approach overcomes the disadvantages in both cost and rigour of the standard approach of translation and back-translation. While widely accepted, backtranslation as a quality check has been shown to identify problems in translation that are not there and miss translation concerns that may be important to address. ${ }^{20}$ In addition to the substantial cost of back-translation, these shortcomings highlight the need for a more pragmatic approach such as the one described in this article.

Second, this approach to transcription and translation has pragmatic advantages in terms of time and cost of data processing. Professional transcription and translation services can take weeks to turn recordings into useable transcripts. This approach not only reduced that time to days but also had the added advantage of incorporating important data and annotations from the interviews, such as non-verbal communication and notes about translated meanings, in one place. This streamlining of data into a single annotated transcript for each interview helped to increase efficiency in the analysis steps and retained the preliminary interpretation that occurs during the conduct and debriefing of semistructured interviews. Rather than reviewing two or three documents per interview, the researchers had a single 'master' transcript for each interview.

Third, this approach has the advantage of improving inclusivity both in the study sample and on the research team. Standard professional transcription and translation approaches outsource these steps to people outside the local community who are unfamiliar with the research question and procedures. Rather, this approach further immersed the research assistants in the data. In addition, having trusted local community members with experience in the super-diverse communities of interest increased the research team's ability to engage multiple subpopulations from the community, ensuring a more inclusive sample than might otherwise have been possible. Finally, each of the four research assistants reported that the experience increased their capacity for working in and supporting the health of their communities. In fact, three of the four used the experience to help further their education in health-related fields.

However, this approach also has some potential limitations. First and foremost, an incomplete use of the co-researcher approach may have limited the research assistants' ability to contribute to the conception of the study and thus the overall findings. In this case example, the coresearchers were brought in after the research question and design were developed. They also had limited roles in revising-rather than codeveloping-interview questions and in interpretation of study findings. A more complete use of the coresearcher approach from the early stages of the research process may have made the overall project more robust.

Second, community coresearchers may have biases or 'blind spots' as interviewers and annotators that the researchers cannot effectively perceive or evaluate. Because the case example used here only had one co-researcher per community and collected deidentified data from participants, we were unable to have coresearchers achieve consensus in their transcription, annotation and interpretation of data, nor were we able to conduct member checking with interview participants. Future applications of this approach should consider having multiple coresearchers per community, conducting member checking, and other measures to ensure validity such as triangulation with other data sources.

Ultimately, the approach we describe in this article added information that increased the validity of interpretation and analysis and improved researchers' understanding of primary care experiences for young adult refugees in a superdiverse community. The combination of components in this approach-community engaged, multilingual coresearchers, translation and transcription, annotation with non-verbal and cultural data-resulted in additional data that improved the richness of information available and increased the validity of interpretation and analysis in this study.

\section{Methodological and practical application}

We offer the following practical advice in the areas of staffing, transcription and annotation for researchers who are conducting qualitative research with using this approach. First, researchers who are conducting qualitative research with communities where language and culture could make interpretation of data challenging should consider hiring and training bilingual or multilingual research assistants who are also members of the community of interest. Research assistants should also receive training in transcription processes. Teams should agree on an annotation format (eg, colour, italics, brackets, asterisks) for all non-verbatim annotations and consider using various formats to denote different types of data incorporated into the transcript. For example, italicising non-verbal communication and bracketing notes about translated meaning of words consistently across a team can make it easier for the team to make sense of all types of data during analysis. Finally, teams should develop clear procedures for what types of annotations should be included in both field notes and transcribed interviews (eg, cultural and religious references, non-verbal cues, idiomatic phrases) and err on the side of including observations or comments they are unsure are relevant so not to lose potentially valuable information. While it could be true that these annotations introduce some level of interpretation to the transcripts, the annotation formatting procedures should make clear what is 
data directly from participants and what is added through observation of the participations or through interpretation by the research assistants during the interview or transcription and translation processes.

\section{Contribution to family medicine and community health research}

This approach to data processing, specifically focused on translation, transcription and annotation, conducted in collaboration with trained bilingual or multilingual research assistants who are also members of the community of interest can improve the retention of nuance and meaning in qualitative data collection in family medicine and primary care research. Researchers conducting qualitative research with diverse-and especially non-English, non-Spanish speaking-patient populations should consider using these community-engaged data collection and processing approaches to ensure retention of both linguistic and cultural meaning. Finally, additional work should explore the usefulness of similar approaches in other diverse communities.

\section{CONCLUSION}

Family medicine and primary care researchers have a need for approaches and tools to increase the inclusivity of their research samples and capacity to explore qualitative research questions in superdiverse communities. This community-engaged, pragmatic approach to processing interview data provides one approach for family medicine researchers to better understand important primary care research questions in superdiverse communities by retaining and adding information that can enhance the richness of transcribed data and increase the validity of interpretation and analysis.

Contributors SEB designed and conducted the study in which this method and approach was adapted and drafted this manuscript. SEB is the overall content guarantor. JS consulted on the original study, drafted sections of this manuscript, and critically reviewed and revised the manuscript.

Funding The authors have not declared a specific grant for this research from any funding agency in the public, commercial or not-for-profit sectors.

Competing interests None declared.

Patient consent for publication Not applicable.

Ethics approval Study procedures were reviewed and approved as exempt by the Colorado Multiple Institutional Review Board (COMIRB), protocol \#18-1081.

Provenance and peer review Not commissioned; externally peer reviewed. Data availability statement № data are available.

Open access This is an open access article distributed in accordance with the Creative Commons Attribution Non Commercial (CC BY-NC 4.0) license, which permits others to distribute, remix, adapt, build upon this work non-commercially, and license their derivative works on different terms, provided the original work is properly cited, appropriate credit is given, any changes made indicated, and the use is non-commercial. See: http://creativecommons.org/licenses/by-nc/4.0/.

ORCID iD

Sarah E Brewer http://orcid.org/0000-0003-0063-6626

\section{REFERENCES}

1 Vertovec S. Towards post-multiculturalism? Changing communities, conditions and contexts of diversity. Int Soc Sci J 2010;61:83-95.

2 Vertovec S. Super-diversity and its implications. Ethnic and Racial Studies 2007;30:1024-54.

3 U.S. Census Bureau. Quick facts: United States, 2019. Available: https://www.census.gov/quickfacts/fact/table/US/PST045219\# PST045219 [Accessed 27 Aug 2020].

4 Blommaert J, Backus A. Superdiverse repertoires and the individual. Multilingualism and multimodality. Brill Sense, 2013: 9-32.

5 Robinson D. The neighbourhood effects of new immigration. Environment and Planning A 2010;42:2451-66.

6 Larkin PJ, Dierckx de Casterlé B, Schotsmans P. Multilingual translation issues in qualitative research: reflections on a metaphorical process. Qual Health Res 2007;17:468-76.

7 Ozolins U, Hale S, Cheng X, et al. Translation and backtranslation methodology in health research - a critique. Expert Rev Pharmacoecon Outcomes Res 2020;20:69-77.

8 Gibb R, Danero Iglesias J. Breaking the silence (again): on language learning and levels of fluency in ethnographic research. Sociol Rev 2017;65:134-49.

9 Ş B. Moving in-between: the interpreter as ethnographer and the interpreting-researcher as anthropologist. Meta: Journal des traducteurs 2004;49:805-21.

10 Chidarikire S, Cross M, Skinner I, et al. Navigating nuances of language and meaning: challenges of cross-language ethnography involving Shona speakers living with schizophrenia. Qual Health Res 2018;28:927-38.

11 Brewer SE. Patterns of primary care use among young adult refugees resettled in Colorado. J Immigr Minor Health 2021;23:1--10.

12 Brewer SE. Factors and experiences of primary care use among young adult refugees. Available from Dissertations \& Theses @ University of Colorado System; ProQuest Dissertations \& Theses A\&I. (2322130887) 2019.

13 Brewer SE. Primary care experiences of young adult refugees after resettlement. 2019 North American Refugee Health Conference, Toronto, CA, 2019.

14 Westfall JM, Zittleman L, Felzien M, et al. Institutional review board training when patients and community members are engaged as researchers. Fam Pract 2017;34:301-4.

15 Israel BA, Coombe CM, Cheezum RR, et al. Community-Based participatory research: a capacity-building approach for policy advocacy aimed at eliminating health disparities. Am J Public Health 2010;100:2094-102.

16 Minkler M. Community-based research partnerships: challenges and opportunities. J Urban Health 2005;82:ii3-12.

17 Semaan S. Time-space sampling and respondent-driven sampling with hard-to-reach populations. Methodological Innovations Online 2010;5:60-75.

18 Muhib FB, Lin LS, Stueve A, et al. A venue-based method for sampling hard-to-reach populations. Public Health Rep 2001;116 Suppl 1:216-22.

19 Atkinson R, Flint J. Accessing hidden and hard-to-reach populations: Snowball research strategies. Social research update 2001;33:1-4.

20 Behr D. Assessing the use of back translation: the shortcomings of back translation as a quality testing method. Int J Soc Res Methodol 2017;20:573-84. 